\title{
Rúbrica socioformativa para validar el constructo "gestión del conocimiento" durante el desarrollo de proyectos formativos con estudiantes de ingeniería
}

\author{
Socioformative Rubric to Validate the "Knowledge Management" Construct during the Development \\ of Training Projects with Engineering Students
}

\section{Paula Flora Aniceto-Vargas ${ }^{1}$, Josemanuel Luna-Nemecio ${ }^{2}$, María de Lourdes Rodríguez-Peralta ${ }^{3}$}

\author{
${ }^{1}$ Instituto Politécnico Nacional, MÉXICO \\ https://orcid.org/0000-0002-6634-6210 | paniceto@ipn.mx \\ ${ }^{2}$ Centro Universitario CIFE, MÉXICO \\ https://orcid.org/0000-0002-6850-3443 | josemanuelluna@cife.edu.mx \\ 3Instituto Politécnico Nacional, MÉXICO \\ https://orcid.org/0000-0002-6634-6210 | mlrodriguezp@ipn.mx
}

Recibido 25-03-2020, aceptado 22-06-2020.

\begin{abstract}
Resumen
Una de las necesidades que se tiene en escuelas de nivel superior, es la falta de instrumentos confiables para realizar evaluaciones objetivas de los desempeños que los estudiantes desarrollan. En ese contexto, en este artículo, se reportó el proceso que se llevó a cabo para validar el constructo "gestión del conocimiento" (GC), en una rúbrica socioformativa, diseñada para evaluar la "GC" en estudiantes de nivel superior en el área de Ingeniería, durante el desarrollo de proyectos formativos y se determinó su confiabilidad. Se aplicó un estudio metodológico descriptivo de validez y confiabilidad de constructo "GC" a través del análisis factorial exploratorio, se usó el software estadístico SPSS. Este estudio, se llevó a cabo con una muestra población objetivo de 152 estudiantes de la Carrera de Ingeniería en Comunicaciones y Electrónica (ICE), de la Escuela Superior de Ingeniería Mecánica y Eléctrica (ESIME), Unidad Culhuacán (UC), del Instituto Politécnico Nacional (IPN). La rúbrica Socioformativa resultó con validez de contenido, determinada a través del cálculo de la $\mathrm{V}$ de Aiken, con valores superiores a 0.8 , en la mayoría de las variables, con aportaciones de juicio de expertos. La fiabilidad del instrumento se determinó a través de dos métodos: el alfa de Cronbach (grupo piloto) y a través del análisis factorial exploratorio (muestra población objetivo), resultando en ambos métodos, valores mayores de 0.8 . Se concluye: la rúbrica socioformativa, con la que se pretende evaluar el constructo "gestión del conocimiento", muestra validez de constructo aceptable y alta confiabilidad para ser aplicado en el contexto descrito.
\end{abstract}

Palabras clave: Constructo, evaluación, gestión del conocimiento, proyectos formativos, rúbrica socioformativa, validez.

\begin{abstract}
One of the needs of higher-level schools is the lack of reliable instruments to carry out objective evaluations of the performance that students develop. In that context, this paper reported the process implemented to validate the construct "Knowledge Management" (GC - For its acronym in Spanish) in a socio-formative rubric designed to evaluate the "GC" on University Engineering students during the development of formative projects and its reliability was determined. A descriptive methodological study of "GC" construct validity and reliability through the exploratory factor analysis, using the SPSS statistical software. This study was applied to a sample target population of 152 students of Electronics and Communications Engineering (ICE), of the Escuela Superior de Ingeniería Mecánica y Eléctrica (ESIME), Culhuacán Campus (UC), at Instituto Politécnico Nacional (IPN). The socio-formative rubric resulted in content validity, determined through the calculation of the Aiken's $\mathrm{V}$ with values over 0.8 in most of the variables, with judgment contributions of experts. The instruments' reliability was determined through two methods: Cronbach's alpha (Pilot group) and the Exploratory Factor Analysis (sample target population), resulting in both methods values over 0.8. it is concluded: The socioformative rubric, with which it is intended to evaluate the construct "knowledge management", shows acceptable construct validity and high reliability to be applied in the described context.
\end{abstract}

Index terms: Construct, evaluation, knowledge management, training projects, socioformative rubric, validity. 


\section{INTRODUCCIÓN}

En escuelas de nivel superior, en el área de ingeniería; existe la necesidad de implementar instrumentos diseñados, validados y confiables, para evaluar objetivamente el proceso de gestión del conocimiento "GC" durante el desarrollo de proyectos formativos, como fuente de construcción de los aprendizajes y desempeños que los estudiantes desarrollan durante su formación académica. En ese sentido, [1] considera que la evaluación es un proceso que no se puede aislar de las personas que continuamente están buscando mejorar, tanto su entorno, como de sí mismas, es por ello, que se considera un tema relevante que debe ser atendido desde la experiencia del docente, de los funcionarios de una Institución educativa, por los mismos estudiantes, los empresarios, desde la familia, o cualquier otro rol que se desempeñe como miembro de una sociedad.

Desde la socioformación se propone la evaluación socioformativa, ésta ha ido incrementando su importancia por considerar en sus procesos los aspectos metodológicos con enfoque en la formación integral en donde se ha iniciado con la sistematización y aplicación de instrumentos de evaluación, como son las rúbricas socioformativas, éstas se han llevado a las aulas a través de la evaluación diagnostica, continua y sumativa articulando la autoevaluación, coevaluación y la heteroevaluación con enfoque colaborativo [2]. Posibilitando el mejoramiento continuo, tomando como base a la taxonomía socioformativa que considera los 5 niveles de desempeño: preformal, receptivo, resolutivo, autónomo y estratégico [3]. Desarrollando el talento en los estudiantes a través de la retroalimentación en la evaluación, co y heteroevaluación en la resolución de problemas de contexto y análisis de evidencia [4]. [2] Dar la oportunidad de visualizar y mejorar el nivel de dominio y desempeño en los estudiantes en una gama de indicadores y descriptores con niveles de dominio. Contribuir en la mejora de los desempeños a través de una evaluación metacognitiva, que los estudiantes se preparen para la vida, con ética, valores, para la contribución en la mejora del planeta en todos los ámbitos: educativo, social, político, económico y sostenibilidad [5].

Haciendo referencia al constructo "gestión del conocimiento" (GC), [6] [7] lo describen como una capacidad para organizar e innovar en otros conocimientos. [8] lo considera como una función que proyecta, dispone e interviene en el movimiento del conocimiento que se genera en una empresa en función con las actividades y con el entorno para crear nuevos conocimientos. [9] la concibe, como capacidad colaborativa para adquirir y generar conocimiento para ser aplicado para un bien común ante la sociedad. [6] resume diciendo que la GC, es integralmente organizar la información y el conocimiento de una empresa o en una persona.

Etimológicamente, [4] comparten la definición de gestión del conocimiento, quienes consideran, que está compuesta de los términos gestión y conocimiento; en donde, gestión, hace referencia a las acciones o trámites que se realizan para conseguir o resolver algo. Del latín gestio, acción y consecuencia de administrar o gestionar algo [10], ahí mismo se define conocimiento, como acción o efecto de conocer, relacionado con el entendimiento, inteligencia, razón natural y a las facultades sensoriales del hombre, su noción y concepto se encuentra principalmente inscrito con la ciencia y/o sabiduría.

Desde el enfoque socioformativo, se considera que, a partir de la GC, las personas busquen, seleccionen, organicen, analicen, apliquen idóneamente, adopten y desarrollen conocimiento, para que identifiquen, interpreten, argumenten y solucionen problemas del contexto, aplicando diferentes saberes, actuando con ética y trabajando colaborativamente [2], en el desarrollo de proyectos formativos. La evaluación del constructo (GC), se hace a través de un instrumento confiable y validado en su contenido a través de juicio de expertos. En la actualidad el juicio de expertos es considerado un proceso en donde el investigador comparte el instrumento con personas especialistas en el tema y que son reconocidos por otros como expertos calificados en él. La finalidad es recibir información certera, reflexiones y valoraciones del instrumento [11], [12].

Con respecto a los proyectos formativo, [3] los describen, como una metodología que se aplica para desarrollar y evaluar competencias; para su implementación es necesario articular acciones para resolver problemas de contexto, bajo la colaboración, ética, valores y la cocreación del conocimiento, buscando que los estudiantes adquieran aprendizajes útiles en la sociedad del conocimiento, para su evaluación es necesario involucrar tres etapas: al inicio del proceso, (evaluación diagnostica), durante el proceso (evaluación formativa) y al final del proceso (evaluación para acreditar). Para evitar que haya subjetividad en la evaluación al incorporar en el proceso estos tipos de evaluación, se recomienda la implementación de rúbricas socioformativas como instrumentos que evalúen la GC, durante el desarrollo de proyectos formativos. Para ello, se cuenta con una rúbrica socioformativa, con validez 
de contenido, realizada en otra investigación por los mismos autores, su diseño se hizo bajo el principio de una escala tipo Likert, considerando la taxonomía socioformativa para la redacción de los niveles de desempeño.

Con respecto a la validez del constructo "GC" en la rúbrica Socioformativa, se aborda el proceso descriptivo del análisis factorial exploratorio, con la finalidad de dar cuenta sobre que variables cargan en mayor proporción para cada factor de los considerados en el diseño de la rúbrica socioformativa, con la finalidad de evaluar objetivamente la gestión del conocimiento en estudiantes del área de ingeniería, durante el desarrollo de proyectos formativos. Este análisis abona al investigador, información sobre las variables que son relevantes a considerar por cada dimensión o factor, para validar el constructo "GC" en la rúbrica socioformativa en cuestión. En función del análisis factorial confirmatorio, en este estudio no se trastocó información al respecto, con la finalidad de dejar huecos en la investigación y dar la oportunidad de realizar o continuar con este estudio en otro momento o por otros investigadores.

El software estadístico SPSS, es una herramienta que aporta elementos para realizar análisis estadístico de muestras significativas de una población a la que está dirigida la investigación. Entre los análisis, que se encuentran en su repertorio están, el análisis factoría exploratorio. [13] lo consideran como uno de los análisis estadísticos más usados en la investigación en ciencias sociales, para determinar el número apropiado de factores o componentes y descubrir cuales variables son indicadores razonables de dichos factores, no se especifica de antemano entre las variables y número de factores o componentes. Así también el análisis de factor confirmatorio, se aplica con la finalidad de evaluar discrepancias metodológicas y de medición psicométricas que pudiera tener el instrumento, además es el método relevantemente aplicado para evidenciar validez de constructo, puesto que informa sobre la estructura interna del instrumento de medida [14].

A partir del software estadístico SPSS, se confirma la fiabilidad del instrumento [15]. Se determinó mediante la aplicación de la rúbrica socioformativa a un grupo piloto de 54 estudiantes de diferentes semestres de la carrera de Ingeniería en Comunicaciones y Electrónica (ICE), de acuerdo con el mapa curricular vigente. La aplicación de la rúbrica socioformativa la hicieron 9 docentes de los mismos semestres y de la misma carrera a la que pertenecen los estudiantes considerados en el proceso, previo a la aplicación de la rúbrica, los estudiantes desarrollaron colaborativamente un proyecto formativo, asesorado y evaluado por los mismos docentes, quienes llevaron el proceso de aplicación. Cabe mencionar que la aplicación del instrumento se hizo a través de un formulario diseñado con Google, se puede observar para más detalle en el enlace siguiente https://docs.google.com/forms/d/1mEg01_djKFBb86gN0n8y1EHRYZJTufZzMRYgLhDC1Xw/edit.

Con los resultados de la aplicación, se generó una matriz con los valores de las variables involucradas, asignadas de acuerdo con los niveles de desempeño, estos dan la oportunidad al docente de medir el progreso de los estudiantes, durante el proceso de aprendizaje. Se muestra una semblanza de las características observables para cada nivel de desempeño desde la socioformación [3] (véase Tabla 1).

Como resultado de la aplicación del software estadístico SPSS, en el cálculo del alfa de Cronbach (un índice de confiabilidad), se tienen valores superiores a 0.8. [16]; [17] La evaluación de los aprendizajes en escuelas de ingeniería, que se realice aplicando rubricas socioformativas, es un tema relevante puesto en discusión por los autores de este artículo, mismo que se ha tratado por varios autores, debido a la importancia que enmarca en el proceso de aprendizaje y mejora continua de los estudiantes. [3] hacen referencia que, en el modelo educativo basado en competencias, la evaluación es el eje central de la formación académica por la importancia que tiene en el proceso de aprendizaje de los estudiantes.

TABLA 1

NIVELES DE DESEMPEÑO DESDE LA SOCIOFORMACIÓN

\begin{tabular}{|c|c|c|c|c|}
\hline Preformal & Receptivo & Resolutivo & Autónomo & Estratégico \\
\hline $\begin{array}{l}\text { Desempeño tiene ideas } \\
\text { o acercamiento al } \\
\text { tema o problema, sin } \\
\text { claridad conceptual ni } \\
\text { metodológica. }\end{array}$ & $\begin{array}{l}\text { Desempeño con } \\
\text { nociones de las } \\
\text { cosas y reproducción } \\
\text { de herramientas sin } \\
\text { comprensión. }\end{array}$ & $\begin{array}{l}\text { Desempeño con } \\
\text { conceptos y } \\
\text { herramientas con } \\
\text { comprensión y } \\
\text { problemas sencillos. }\end{array}$ & $\begin{array}{l}\text { Desempeño con } \\
\text { autonomía cognitiva } \\
\text { y abordaje de } \\
\text { problemas con } \\
\text { análisis y } \\
\text { argumentación. }\end{array}$ & $\begin{array}{l}\text { Desempeño con base } \\
\text { en estrategias, la } \\
\text { creatividad, el antro } \\
\text { poético y el abordaje } \\
\text { de problemas inter y } \\
\text { transdisciplinarios. }\end{array}$ \\
\hline
\end{tabular}


El problema que se enmarca en el contexto descrito, es la falta de instrumentos de evaluación, validados en su contenido, confiables y que el constructo GC sea viable para ser aplicado en la población objetivo, para este caso específico, estudiantes de la Carrera de ICE, del Instituto Politécnico Nacional, Se observa que los docentes, al menos los invitados a participar y que no se involucraron, se muestran ajenos y con falta de conocimientos previos sobre las metodologías de aplicación de la estrategia de proyectos formativos, el constructo GC y la aplicación de instrumentos durante la evaluación de los aprendizajes. Se visualiza la necesidad de lograr que los estudiantes se apropien del conocimiento de manera colaborativa, resuelvan problemas de su contexto, que contribuyan en la economía del país, en la sostenibilidad del ambiente en todo el planeta, se formen para la vida a través de acciones éticas, responsables e idóneas [5].

En este trabajo se presenta las características propias de la rúbrica socioformativa que se diseñó para evaluar la gestión del conocimiento durante el desarrollo de proyectos formativos, el conocimiento que tanto los estudiantes como los docentes deben tener de ella y su aplicación como instrumento de evaluación en el contexto descrito, así también se comparten los procesos previos a la validación del constructo, estos son la validación de contenido y confiabilidad.

Con base en lo descrito, se consideran las siguientes metas que se tienen previstas en este documento:

1. Realizar una investigación en la literatura correspondiente y en función de ella crear preguntas o variables integradas en dimensiones (Diseño de la rúbrica socioformativa) que correspondan a la evaluación de la gestión del conocimiento que se lleva a cabo durante el desarrollo de proyectos formativos, por estudiantes de la carrera de ICE.

2. Aplicar la rúbrica socioformativa a una población objetivo de la carrera de ICE y tener resultados sobre la interpretación de las variables por cada dimensión.

3. Usar los resultados de la aplicación de la rúbrica socioformativa, para realizar el análisis factorial exploratorio con el programa estadísticos SPSS y conocer si el modelo propuesto corresponde con el resultado del análisis.

4. Analizar los resultados del factor exploratorio, para saber cómo la población objetivo, está interpretando las variables, cuales se relacionan con cuales y determinar la fiabilidad del instrumento y la validación de constructo GC durante el desarrollo de proyectos formativos.

\section{DESARROLLO}

\section{A. Tipo de estudio}

Se aplicó un estudio metodológico descriptivo de validez y fiabilidad de constructo "gestión del conocimiento" a través del análisis factorial exploratorio, en donde se aplicó el programa estadístico SPSS (AFE). [13] ellos lo considera un método estadístico que es usado con frecuencia en la investigación en ciencias sociales y una técnica en la que los investigadores deben aplicar criterios al momento de la ejecución de dicho análisis, por su parte [18] define el análisis factorial exploratorio (AFE) [Exploratory Factory Analysis; EFA] "como una técnica estadística que permite explorar con mayor precisión las dimensiones subyacentes, constructos o variables latentes de las variables observadas, es decir, las que observa y mide el investigador" [18, p. 1] para la aplicación del AFE, es necesario considerar un tamaño de muestra no menor de 50 participantes, la sugerencia es que el tamaño de la muestra sea mayor de 100, lo más recomendado, es considerar el rango que está entre 300 y 400, Ahí mismo se hace referencia a los casos por variables (Ratio de observaciones), considerando que no hay un consenso unificado, se puede discurrir que haya por lo menos 10 a 15 casos por variable. Este estudio, se aplicó en el semestre agosto-diciembre de 2019, participaron en el proceso 172 estudiantes del área de ingeniería a la cual se le llamó población objetivo, que se integró en 41 proyectos formativos, mismos que fueron evaluados por 11 docentes de las asignaturas de Calculo integral y diferencial de $1^{\circ}$ semestre, Química Aplicad de $2^{\circ}$ semestre, Humanidades V de $5^{\circ}$ semestre, Electrónica digital de $6^{\circ}$ semestre y diseño y administración de redes de $9^{\circ}$ semestre. 


\section{B. Participantes}

En la Tabla 2 y Tabla 3 se observan las características de los participantes.

TABLA 2

DATOS SOCIODEMOGRÁFICOS DE LA POBLACIÓN OBJETIVO, TOMADOS DE LA ENCUESTA SOCIODEMOGRÁFICA.

\begin{tabular}{l|l|l|}
\hline Sexo & hombres $\mathbf{7 3 \%}$ & mujeres 27\% \\
\hline Promedio de edad & 24 años \\
\hline Estado civil & solteros \\
\hline Promedio de años de estudio entre & Entre 12 y 18 \\
\hline Condiciones económicas & Media - Alta \\
\hline Zona de residencia & Ciudad de México y Estado de México \\
\hline Situación laboral & No trabajan \\
\hline
\end{tabular}

TABLA 3

DATOS SOCIODEMOGRÁFICOS DE DOCENTES PARTICIPANTES EN LA APLICACIÓN DE LA RÚBRICA SOCIOFORMATIVA, TOMADOS DE ENCUESTA.

\begin{tabular}{ll}
\hline Sexo & hombres $\mathbf{4 5 \%}$ \\
\hline Promedio de edad & mujeres 55\% \\
\hline Estado civil & Caños \\
Promedio de años de estudio & 25 \\
Condiciones económicas & Media - Alta \\
Zona de residencia & Ciudad de México y Estado de México \\
Situación laboral & Buena \\
\hline
\end{tabular}

\section{Instrumentos}

Se tiene la rúbrica socioformativa que fue diseñada con formato de escala tipo Likert, contiene 8 ítems (variables), con sus niveles de desempeño, redactados bajo las características de la taxonomía socioformativa, distribuidos en tres dimensiones, misma que evalúan el constructo "gestión del conocimiento", fue validada en contenido y fiabilidad [19] Fue diseñada para ocuparla en la evaluación del constructo "gestión del conocimiento" en estudiantes de ingeniería (entre 18-25 años aproximadamente), durante el desarrollo de proyectos formativos. La rúbrica socioformativa se diseñó con formato de cuestionario de Google, así se trabajó para todos los procesos de evaluación. Se comparte el enlace para su consulta:

https://docs.google.com/forms/d/1OG94EJFcB6vd0LNo9QqVRJvCVcNX3pnstlJ9Jyv-ie0/edit

\section{Método}

\section{1) Diseño del instrumento}

Se hace una semblanza del proceso que se siguió previamente para llegar a la Rubrica socioformativa del constructo "gestión del conocimiento". Después de haber hecho revisión de la literatura en bases de datos indexadas: Redalyc, Scielo, Conricyt y Google Académico, para tener artículos relacionados con el tema y siguiendo los procedimientos de construcción y evaluación de instrumentos cuantitativos y su forma de aplicación [11], [15], [20].

Se redactaron 8 ítems (variables), que se agruparon en tres dimensiones (factores), con escala tipo Likert, en base a la taxonomía socioformativa: 1. Identificación de los requerimientos de información, 2. Estrategias para el procesamiento de la información, 3. Aplicación de conocimiento con calidad y pertinencia para resolver el problema. 


\section{a) Mejoramiento del instrumento}

Los ítems y dimensiones se sometieron a una revisión por un grupo de 4 expertos en diseño y redacción de instrumentos, con sus observaciones y sugerencias se mejoró de manera general, tanto el orden como la ubicación de los ítems en las diferentes dimensiones que tiene el instrumento.

\section{b) Validez de contenido}

Se hizo a través de juicio de expertos, para ello participaron 12 docentes de diferentes escuelas del Instituto Politécnico Nacional, de las áreas de Ingeniería, Sociales y Administración, con experiencia en diseño y evaluación de instrumentos. Sus recomendaciones sirvieron para tener un instrumento pertinente y con buena redacción, se hizo el análisis del cálculo de la $\mathrm{V}$ de Aiken teniendo buenos resultados, en donde un valor aproximado a 1, que significa buena concordancia entre las respuestas de los jueces [12].

\section{c) Confiabilidad del instrumento}

Está referida a la consistencia interna entre los ítems, [21] hacen referencia en su investigación que un valor total del alfa de Cronbach de 0.97 es un buen resultado del coeficiente de confiabilidad. En la investigación previa, [22] para la determinación del alfa de Cronbach, la rúbrica Socioformativa se aplicó a un grupo piloto integrado por 54 estudiantes de la Carrera de Ingeniería en Comunicaciones y Electrónica (ICE), del Instituto Politécnico Nacional (IPN) de la Ciudad de México de los semestres $2^{\circ}, 5^{\circ}, 6^{\circ}, 8^{\circ}$ y $9^{\circ}$ respectivamente (grupo piloto). Estos estudiantes durante el tiempo de la intervención en la asignatura correspondiente trabajaron colaborativamente la gestión del conocimiento en el desarrollo de un proyecto formativo.

\section{d) Validez de constructo}

Finalmente, la validez de constructo "gestión del conocimiento", se hizo a través de la aplicación de la rúbrica Socioformativa a la población objetivo, los resultados se evaluaron a través de la técnica de análisis factorial exploratorio (AFE), aplicando el método de componentes principales y rotación Varimax [23], [13], se comprobó la fiabilidad del instrumento con la determinación del alfa de Cronbach entre todos los ítems del instrumento y de manera específica para cada dimensión, debe tener valores aproximados a 1.0.

Antes de realizar el análisis factorial, se necesita calcular y analizar la matriz de correlaciones o covarianzas de las variables para comprobar si los datos tienen las características apropiadas para llevar a cabo el análisis, con esa mira, se determina el test de esfericidad de Bartlett y el índice de medida de adecuación de la muestra KMO [24] para estos casos, los rangos de valores para estas características son 0.000 y valores que pueden ir desde 0.7 y hasta 1.0 respectivamente. De la misma manera se debe considerar el tamaño de muestra, en ese sentido, la rúbrica socioformativa se aplicó a una muestra de población objetivo de 172 estudiantes, se refiere a [25] quienes consideran que nunca se debe hacer un AFE con una muestra menor de 50 observaciones, de preferencia trabajar con 100 o más unidades o considerar un rango de entre 300 y 400 casos, cabe mencionar que la muestra de la población objetivo fue de 172; sin embargo, en el análisis de eliminación de blancos, la muestra total, bajo la cual se realizó todo el proceso de análisis quedo en 152 casos, no necesariamente se requiere la palabra desarrollo, se pueden denominar las secciones según se requiera y numerar las subsecciones con esta nomenclatura. En la Tabla 4: para el procesamiento de la información y su análisis, se aplicó el software estadístico SPSS versión 24.

TABLA 4

DISTRIBUCIÓN DE LA MUESTRA POR APLICACIÓN DE LA RÚBRICA SOCIOFORMATIVA.

\begin{tabular}{clcc}
\hline Área de formación & \multicolumn{1}{c}{ Asignatura } & Semestre & \% \\
\hline \multirow{4}{*}{ INGENIERÍA } & Calculo diferencial e integral & Primero & 27.61 \\
& Química aplicada & Segundo & 54.60 \\
& Humanidades V & Quinto & 4.90 \\
& Electrónica digital & Sexto & 11.66 \\
& Diseño y administración de redes & Noveno & 1.23 \\
\hline Nota relevante: Los datos para hacer el análisis estadístico correspondiente fueron recogidos al final de la tercera \\
evaluación ordinaria: diciembre de 2019, del semestre agosto - diciembre de 2019 (periodo escolar 1 del 2020). \\
\hline
\end{tabular}




\section{e) Consideraciones éticas}

En todo momento, la participación tanto de docentes como de estudiantes fue consensada y de manera voluntaria, quedando claro que los datos personales serían confidenciales y se les explicó el uso que se le daría.

\section{2) Resultados}

\section{a) Revisión del instrumento}

Las recomendaciones sugeridas por los 4 expertos en diseño y evaluación de instrumentos, después de haber hecho la revisión de manera general sobre ubicación y distribución de los ítems en las diferentes dimensiones, brindó la oportunidad de mejorar la Rúbrica socioformativa en esos aspectos.

\section{b) Validez de contenido}

En la etapa de validación de contenido, realizada por juicio de expertos, con las aportaciones se realizó un análisis de los datos descriptivos de acuerdo con el coeficiente de la $V$ de Aiken en función de la redacción y pertinencia. Los resultados del cálculo del coeficiente de la $V$ de Aiken brindaron la oportunidad de mejorar las variables en los aspectos recomendados, estos se describen en la Tabla 5.

TABLA 5

RESULTADOS DE VALIDEZ DE CONTENIDO MEDIANTE LA V DE AIKEN.

\begin{tabular}{|c|c|c|c|}
\hline \multirow{2}{*}{ Dimensión } & \multirow[b]{2}{*}{ Ítem } & Redacción & Pertinencia \\
\hline & & \multicolumn{2}{|c|}{$V$ de Aiken } \\
\hline \multirow{3}{*}{$\begin{array}{l}\text { Dimensión 1: } \\
\text { Identificación } \\
\text { de los } \\
\text { requerimientos } \\
\text { de información. }\end{array}$} & $\begin{array}{l}\text { 1. ¿En qué nivel los estudiantes identificaron las necesidades } \\
\text { de la información para resolver el problema de } \\
\text { contexto? }\end{array}$ & 0.83 & 0.70 \\
\hline & $\begin{array}{l}\text { 2. ¿En qué nivel el informe escrito contiene citas y referencias } \\
\text { actuales de la información propuesta para resolver el } \\
\text { problema de contexto? }\end{array}$ & 0.50 & 0.50 \\
\hline & $\begin{array}{l}\text { 3. ¿En qué nivel en el informe escrito se integra la lista de } \\
\text { referencias bibliográficas de fuentes de información } \\
\text { confiable y especializada para resolver el problema de } \\
\text { contexto? }\end{array}$ & 0.70 & 0.83 \\
\hline \multirow{3}{*}{$\begin{array}{l}\text { Dimensión 2: } \\
\text { Estrategias para } \\
\text { el Procesamiento } \\
\text { de la } \\
\text { información. }\end{array}$} & $\begin{array}{l}\text { 4. ¿En qué nivel los estudiantes aplicaron estrategias de } \\
\text { selección y sistematización de la información? }\end{array}$ & 0.83 & 0.83 \\
\hline & $\begin{array}{l}\text { 5. ¿En qué nivel los estudiantes emplean estrategias para el } \\
\text { trabajo colaborativo? }\end{array}$ & 0.83 & 0.83 \\
\hline & $\begin{array}{l}\text { 6. ¿En qué nivel los estudiantes presentan evidencias de trabajo } \\
\text { colaborativo, realizado de manera presencial y a } \\
\text { distancia para resolver el problema de contexto? }\end{array}$ & 0.70 & 0.83 \\
\hline \multirow{2}{*}{$\begin{array}{l}\text { Dimensión 3: } \\
\text { Aplicación de } \\
\text { conocimiento con } \\
\text { calidad y } \\
\text { pertinencia para } \\
\text { resolver el } \\
\text { problema. }\end{array}$} & $\begin{array}{l}\text { 7. ¿En qué nivel el conocimiento se aplicó con objetividad y } \\
\text { pertinencia en la resolución del problema? }\end{array}$ & 0.83 & 0.83 \\
\hline & $\begin{array}{l}\text { 8. ¿En qué nivel los estudiantes gestionan conocimiento } \\
\text { pertinente para resolver el problema de contexto? }\end{array}$ & 0.5 & 1.00 \\
\hline
\end{tabular}

Nota: Los ítems en donde resultó el cálculo de la $\mathrm{V}$ de Aiken menor de 7, se mejoraron en función de las recomendaciones de los jueces expertos. 


\section{c) Fiabilidad del instrumento}

Con respecto a la confiabilidad, el análisis estadístico realizado con el programa SPPS, resultó un valor del alfa de Cronbach de 0.85 , lo que significa que el instrumento es confiable, los ítems están altamente correlacionados entre sí (véase Tabla 6).

TABLA 6

CÁlCUlO DEL ALFA DE CRONBACH DE LA APLICACIÓN AL GRUPO PILOTO CON DATOS DE ANÁLISIS FACTORIAL EXPLORATORIO (SPSS VERSIÓN 24).

\section{Estadísticas de fiabilidad}

Alfa de Cronbach

Alfa de Cronbach basada en elementos estandarizados

$N$ de elementos

\section{.851}

.857

8

\section{Estadísticas de elemento}

\begin{tabular}{|c|c|c|c|}
\hline & Media & Desviación estándar & $N$ \\
\hline VAR00001 & 4.1818 & .95799 & 22 \\
\hline VAR00002 & 3.5455 & 1.22386 & 22 \\
\hline VAR00003 & 3.4091 & .95912 & 22 \\
\hline VAR00004 & 3.3636 & 1.25529 & 22 \\
\hline VAR00005 & 3.6364 & 1.25529 & 22 \\
\hline VAR00006 & 3.7273 & .76730 & 22 \\
\hline VAR00007 & 4.2727 & .98473 & 22 \\
\hline VAR00008 & 3.9091 & .75018 & 22 \\
\hline
\end{tabular}

Se aplicó la encuesta de satisfacción del instrumento a los participantes, de donde resultó que el grado de comprensión de las instrucciones del instrumento, el grado de comprensión de las preguntas o ítems, el grado de satisfacción con el instrumento y el grado de relevancia de las preguntas, de manera general, está entre bueno y excelente, los valores sombreados en la Tabla 7 dan indicadores sobre la buena calidad del instrumento en función de la comprensión de las instrucciones, las preguntas, satisfacción y relevancia.

TABLA 7

GRADO DE COMPRENSIÓN Y DE ACEPTACIÓN DE LA RÚBRICA SOCIOFORMATIVA PARA EVALUAR LA GESTIÓN DEL CONOCIMIENTO DURANTE EL DESARROLLO DE PROYECTOS FORMATIVOS, APLICADO A UN GRUPO PILOTO DE ESTUDIANTES DE LA ESIME UC DEL IPN.

\begin{tabular}{|c|c|c|c|c|}
\hline Encuesta de satisfacción & Bajo & Aceptable & Bueno & Excelente \\
\hline $\begin{array}{l}\text { ¿Cuál fue el grado de comprensión de las instrucciones del } \\
\text { instrumento? }\end{array}$ & & 4.5 & 68.2 & 27.3 \\
\hline ¿Cuál fue el grado de comprensión de las preguntas o ítems? & & 13.6 & 59.1 & 27.2 \\
\hline ¿Cuál fue el grado de satisfacción con el instrumento? & & & 68.2 & 31.8 \\
\hline ¿Cuál es el grado de relevancia de las preguntas? & 4.5 & 4.5 & 54.5 & 36.4 \\
\hline
\end{tabular}




\section{3) Validez de constructo: gestión del conocimiento con Análisis factorial exploratorio (AFE)}

Antes de iniciar el proceso del AFE, se hizo una revisión a la base de datos en Excel para eliminar todos aquellos casos en los que no hubo variabilidad en los resultados, para ello se calculó la varianza total y todos aquellos datos que resultaron valores de cero, se eliminaron, después de este proceso, de un total de 172, quedaron 152 casos. Con el SPSS se calculó y analizó la matriz de correlaciones o covarianzas de las variables para comprobar si los datos tienen las características apropiadas para llevar a cabo el análisis. Obsérvese los resultados en la Tabla 8.

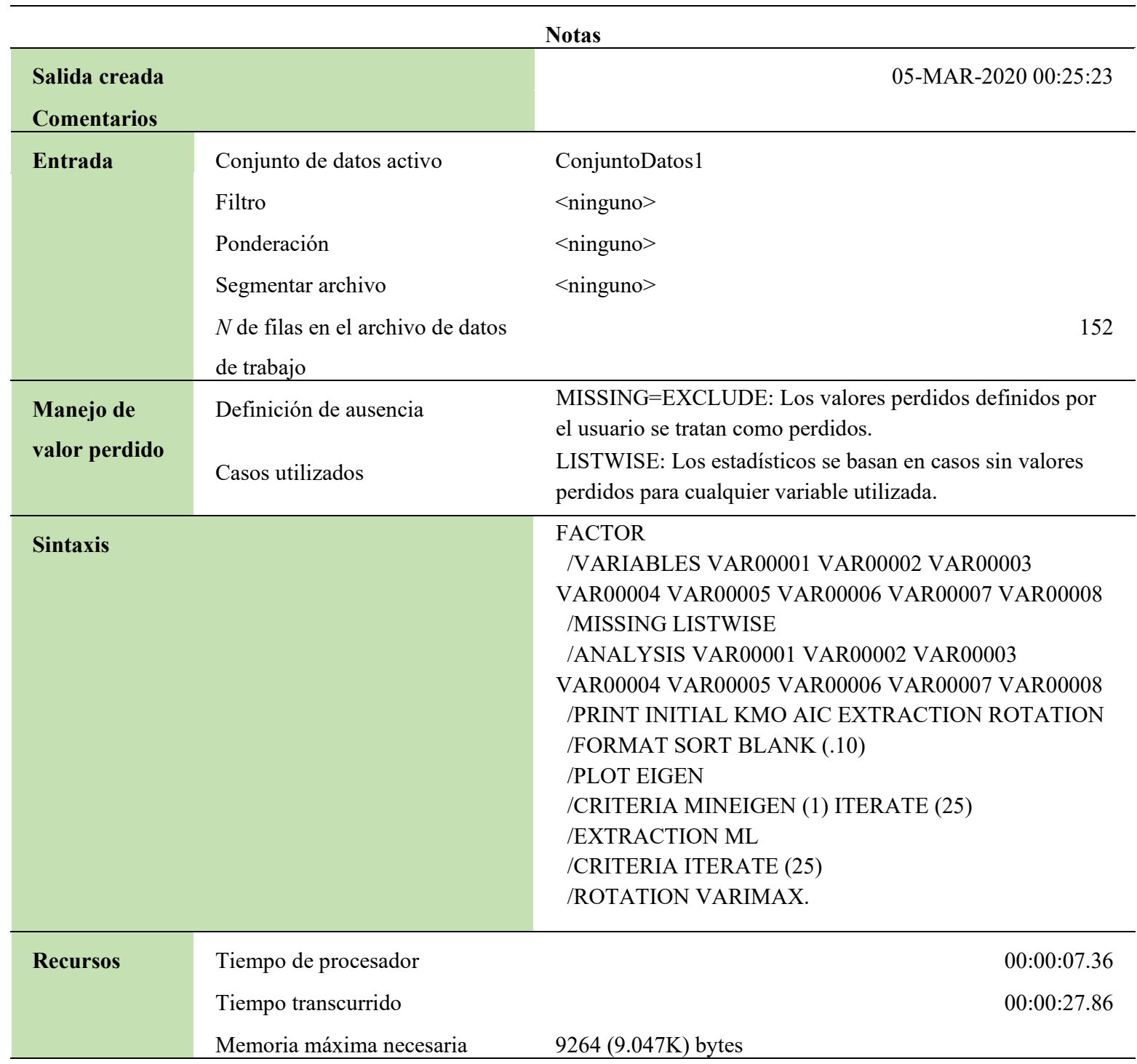

Así también, para comprobar si los datos tienen las características apropiadas para llevar a cabo el análisis factorial exploratorio, se determinó la prueba de esfericidad de Bartlett y el índice de medida de adecuación de la muestra KMO, teniendo los resultados que se muestran en la Tabla 9. Mismos que indican, que los datos son apropiados. 
TABLA 9

PRUEBA DE KMO y DE BARTLETT, CON DATOS DE ANÁLISIS FACTORIAL EXPLORATORIO (SPSS VERSIÓN 24).

\begin{tabular}{|c|c|c|}
\hline \multicolumn{3}{|c|}{ Prueba de KMO y Bartlett } \\
\hline \multicolumn{2}{|c|}{ Medida Kaiser-Meyer-Olkin de adecuación de muestreo } & .803 \\
\hline \multirow[t]{3}{*}{ Prueba de esfericidad de Bartlett } & Aprox. Chi-cuadrado & 781.948 \\
\hline & $\mathrm{gl}$ & 28 \\
\hline & Sig. & .000 \\
\hline
\end{tabular}

Continuando con los resultados del análisis factorial exploratorio, se tiene la matriz factorial y matriz factorial rotado, en donde se observa el número de factores y la contribución de cada variable en los factores o componentes (véase Tabla 10).

TABLA 10

MATRIZ FACTORIAL Y FACTOR DE MATRIZ ROTADO, CON DATOS DE ANÁLISIS FACTORIAL EXPLORATORIO (SPSS VERSIÓN 24).

\begin{tabular}{lcc}
\hline \multicolumn{3}{c}{ Matriz factorial } \\
\hline & 1 & 2 \\
\hline & .848 & -.435 \\
\hline VAR00008 & .807 & \\
VAR00001 & .786 & \\
\hline VAR00002 & .721 & \\
\hline VAR00003 & .693 & .416 \\
\hline VAR00005 & .608 & \\
\hline VAR00004 & .599 & .723 \\
\hline VAR00006 & .609 & \\
\hline VAR00007 &
\end{tabular}

Prueba de bondad de ajuste

\begin{tabular}{rrrrr}
\hline Chi-cuadrado & gl & & Sig. \\
$\mathbf{7 3 . 5 5 5}$ & & 13 & .000 \\
\hline
\end{tabular}

\begin{tabular}{ccc}
73.555 & 13 & .000 \\
\hline Matriz de factor rotado \\
\hline
\end{tabular}

Factor

\begin{tabular}{lcc} 
& 1 & 2 \\
\hline VAR00008 & .937 & \\
VAR00002 & .860 & \\
VAR00006 & .612 & \\
VAR00005 & .551 & .421 \\
VAR00007 & & .944 \\
VAR00004 & & .700 \\
\hline VAR00001 & .490 & .687 \\
\hline VAR00003 & .499 & .534 \\
\hline
\end{tabular}


En un cuarto resultado se tiene la varianza total explicada, en donde se observa un porcentaje acumulado de 72.909 , lo que indica que se tienen las condiciones de realizar una valoración global de los resultados obtenido en la aplicación del análisis, [25] en las ciencias sociales es normal considerar como satisfactorio una solución que represente como mínimo un porcentaje del 60\% de la varianza total (véase Tabla 11).

TABLA 11

VARIANZA TOTAL EXPLICADA, CON DATOS DE ANÁLISIS FACTORIAL EXPLORATORIO (SPSS VERSIÓN 24).

\begin{tabular}{|c|c|c|c|c|c|c|}
\hline \multicolumn{7}{|c|}{ Varianza total explicada } \\
\hline \multirow{2}{*}{ Factor } & \multicolumn{3}{|c|}{ Autovalores iniciales } & \multicolumn{3}{|c|}{ Sumas de extracción de cargas al cuadrado } \\
\hline & Total & $\%$ de varianza & $\%$ acumulado & Total & $\%$ de varianza & $\%$ acumulado \\
\hline 1 & 4.415 & 55.191 & 55.191 & 4.087 & 51.088 & 51.088 \\
\hline 2 & 1.417 & 17.719 & 72.909 & 1.161 & 14.513 & 65.601 \\
\hline 3 & .691 & 8.642 & 81.551 & & & \\
\hline 4 & .574 & 7.173 & 88.724 & & & \\
\hline 5 & .314 & 3.925 & 92.650 & & & \\
\hline 6 & .284 & 3.556 & 96.206 & & & \\
\hline 7 & .175 & 2.194 & 98.399 & & & \\
\hline 8 & .128 & 1.601 & 100.000 & & & \\
\hline \multicolumn{7}{|c|}{ Varianza total explicada } \\
\hline \multirow[t]{2}{*}{ Factor } & \multicolumn{6}{|c|}{ Sumas de rotación de cargas al cuadrado } \\
\hline & & Total & & anza & & umulado \\
\hline 1 & & 2.839 & & & & 35.483 \\
\hline 2 & & 2.409 & & & & 65.601 \\
\hline - & & - & & - & & - \\
\hline
\end{tabular}

Un quinto resultado está relacionado con las comunalidades, la comunalidad indica la proporción de varianza por cada variable que queda explicada por los factores que se han identificado en el análisis, esta información es un buen indicador para establecer hasta qué punto una variable o ítem queda bien representada en el modelo factorial (véase Tabla 12).

TABLA 12. COMUNALIDADES CON DATOS DE ANÁLISIS FACTORIAL EXPLORATORIO (SPSS VERSIÓN 24).

\begin{tabular}{ccr}
\hline \multicolumn{3}{c}{ Comunalidades } \\
\hline & Inicial & Extracción \\
\hline VAR00001 & .730 & .712 \\
\hline VAR00002 & .749 & .768 \\
VAR00003 & .581 & .534 \\
\hline VAR00004 & .571 & .543 \\
VAR00007 & .666 & .893 \\
\hline VAR00008 & .792 & .908 \\
\hline VAR00006 & .488 & .409 \\
\hline VAR00005 & .539 & .480 \\
\hline
\end{tabular}




\section{CONCLUSIONES}

Los desempeños de los estudiantes en escuelas de ingeniería, las estrategias que el docente aplique para llegar a ellos, el reconocimiento por parte del docente, del como aprenden sus estudiantes, el proceso de la gestión del conocimiento(GC), como una capacidad colaborativa para adquirir y generar conocimiento, la aplicación de instrumentos confiables, diseñados y validados para realizar evaluaciones en el aula; son temas relevantes y de pronta atención por los docentes, directivos, familia y para los mismos estudiantes, debido a que son determinantes que implican en la formación académica, para que ésta sea de calidad y haya un desarrollo pleno como resultado del proceso de formación académica [26], [9]. Al respecto del proceso de la GC y cómo llevar a cabo su evaluación; en esta sección se considera que la validación del constructo "GC" y la determinación de la fiabilidad de la rúbrica socioformativa diseñada para evaluar el proceso de la gestión del conocimiento durante el desarrollo de proyectos formativos con estudiantes de nivel superior en el área de ingeniería, es una estrategia necesaria de implementar en el aula, para mejorar los aprendizajes y desempeños de los estudiantes. El resultado del proceso de validación del constructo "GC" a través del análisis factorial exploratorio (AFE), dio la oportunidad de saber que el total de las variables (ítems) consideradas en el diseño de la rúbrica socioformativa en función del constructo "GC" se conservaron, debido a que los valores que resultaron están dentro de rangos establecidos o se inclinan a valores aceptables, lo que indica que las variables consideradas miden sin dificultada el constructo GC en el contexto establecido [13]. El análisis de efecto de los ítems, sobre la validez de constructo permite mencionar que las 8 variables, presentan valor adecuado en la cargan factorial del ítem sobre el factor aceptable al valor de la comunalidad. Los ocho ítems o variables presentan carga superior a 0.5 . El presentar valores de comunalidades dentro de los rangos mayor de 3 , indica que gran parte de la variabilidad del ítem, está siendo explicada por los factores extraídos [18].

A través de los resultados obtenidos en el análisis de fiabilidad con la determinación del alfa de Cronbach [16] realizado a los datos generados de la aplicación de la rúbrica socioformativa a un grupo piloto y a través del análisis factorial exploratorio, permiten afirmar, que se tiene un instrumento con una apropiada consistencia interna entre sus ítems y, en consecuencia, válido para medir el constructo "gestión del conocimiento" (GC) [3].

La aplicación de rúbricas Socioformativas, como instrumento para evaluar el proceso de la GC, durante el desarrollo de proyectos formativos, que brinde a la comunidad involucrada las garantías para ser utilizada en los contextos descritos, es un factor que servirá para asegurar que los niveles de desempeños sean los óptimos y las evaluaciones que se realicen se hagan objetivamente, considerándolo un aporte que en escuelas de Ingeniería se requiere con la finalidad de consolidar estrategias efectivas que aporten en las mejoras de los problemas del contexto descritos [27], [6], [13].

Se puede considerar, en función de los diversos análisis que se realizaron, que pensar en la implementación en el aula de instrumentos de este tipo, puede contribuir en la evaluación del constructo "gestión del conocimiento", durante el desarrollo de proyectos formativos, en donde se ofrecen datos que faciliten la toma de decisión para el diseño del instrumento descrito, accionar puntualmente para el mejoramiento de los desempeños [28].

De manera paralela, considerando las garantías de validez de constructo y fiabilidad que ofrece la rúbrica socioformativa, se piensa que este instrumento, supone una herramienta con valor significativo para futuras investigaciones que deseen profundizar en esta temática. Conocer cómo se perciben las rúbricas socioformativas por los estudiantes del área de ingeniería o de otras áreas de formación y contribuir en la mejora de los desempeños a través de una evaluación metacognitiva, que los estudiantes se preparen para la vida, con ética, valores, para la contribución en la mejora del planeta en todos los ámbitos: educativo, social, político, económico y sostenibilidad [29], [30], [5].

\section{AGRADECIMIENTOS}

A las Autoridades de la Escuela Superior de Ingeniería Mecánica y Eléctrica (ESIME), Unidad Culhuacán (UC), del Instituto Politécnico Nacional (IPN), de la Carrera de Ingeniería en Comunicaciones y Electrónica (ICE), Se agradece y reconoce el interés para que haya evaluaciones objetivas de los aprendizajes que los estudiantes adquieren y por las facilidades brindadas para que se llevara a cabo esta investigación en el interior de la Unidad Académica. A los docentes, de igual manera se agradece el entusiasmo y espíritu de ayuda que manifestaron durante el proceso de aplicación a un grupo piloto y una 
muestra significativa de estudiantes de diferentes semestres de ICE, de la rúbrica Analítica Socioformativa como instrumento de evaluación del constructo "gestión del conocimiento", con la finalidad de corroborar su confiabilidad. A los estudiantes de primero, segundo, quinto, sexto octavo y noveno semestre, se les agradece su participación por haber respondido con honestidad y responsabilidad a la aplicación de la rúbrica analítica Socioformativa, como instrumento de evaluación del constructo "gestión del conocimiento", durante el desarrollo de proyectos formativos.

\section{REFERENCIAS}

[1] J. S. Hernández-Mosqueda, "Procesos de evaluación de las competencias desde la socioformación,” Ra Ximhai, vol. 9, no. 4, Sep. 2013, pp. 11-19, available: https://www.redalyc.org/pdf/461/46129004001.pdf

[2] S. Tobón, Formación integral y competencias: pensamiento complejo currículo, didáctica y evaluación, $3^{\mathrm{a}}$ ed. Bogotá, Colombia: Ecoe, 2010.

[3] S. Cardona, J. Vélez, S. Tobón, "Contribución de la evaluación Socioformativa al rendimiento académico en pregrado," Educar, vol. 52, no.2, pp. 423-447, 2016, available: http://dx.doi.org/10.5565/rev/educar.763

[4] M. F. Ortega-Carbajal, J. S. Hernández-Mosqueda, S. Tobón, "Análisis documental de la gestión del conocimiento mediante la cartografía conceptual,” Ra Ximhai, vol. 11, no. 4, pp. 141-160, Jul. 2015, available: https://www.redalyc.org/pdf/461/46142596009.pdf

[5] J. Luna-Nemecio, Para pensar el desarrollo social sostenible: múltiples enfoques, un mismo objetivo, Mount Dora (USA)/Quito (Ecuador): Kresearch/ Religación, Centro de Investigaciones en Ciencias Sociales y Humanidades desde América Latina, 2020, doi: https://doi.org/10.35766/dss20

[6] I. Cantón, E. Ferrero, "La gestión del conocimiento en revistas de educación,” Educar, vol. 52, no. 2, pp. 401-422, Jul. 2016, doi: http://dx.doi.org/10.5565/rev/educar.757

[7] Á. L. Arboníes, Cómo evitar la miopía en la gestión del conocimiento, Madrid, España: Díaz de Santos, 2011.

[8] E. Bueno, "El gobierno o gestión del conocimiento como estrategia de creación de valor," Cuadernos de Gestión del Conocimiento Empresarial, no. 16, pp. 1-5, Oct. 2009, available: http://www.directivoscede.com/sites/default/files/document/conocimiento/31-07-2013/10cuaderno0000003578.pdf

[9] Medical Law UK. "Home.” Medical Law UK. http://www.unsystemceb.org

[10] RAE, Ortografia de la lengua española, Madrid, España: Espasa, 2010, p. 745.

[11] J. Vázquez- Antonio, S. Tobón, J. M. Vázquez- Antonio, C. E. Guzmán, S. R. Herrera- Meza, “Diseño y validez de contenido de una rúbrica socioformativa para evaluar la tesis de investigación en la Educación Normal," Revista espacios, vol. 39, no. 53, pp. 28, 2018, available: http:/www.revistaespacios.com/cited2017/cited2017-28.html

[12] J. Escobar-Pérez, A. Cuervo-Martínez, "Validez de contenido y juicio de expertos: una aproximación a su utilización," Avances en medición, no. 6, pp. 27-36, 2008, available: http://www.humanas.unal.edu.co/psicometria/files/7113/8574/5708/Articulo3_Juicio_de_expertos_27-36.pdf

[13] D. Frías-Navarro, M. Pascual Soler, "Prácticas del análisis factorial exploratorio (AFE) en la investigación sobre conducta del consumidor y marketing," Suma pedagógica, vol. 19, no. 1, pp. 47-58, Jun. 2012, available: https://www.uv.es/ friasnav/FriasNavarroMarcopsSoler.pdf

[14] M. S. Moscoso, C. Merino-Soto, S. Domínguez-Lara, C. Cecilia, B. Chau, M. Mary-Claux, "Análisis factorial confirmatorio del inventario multicultural de la expresión de la ira y hostilidad," Liberabit, vol. 22, no. 2, pp. 137 152, 2016, available: http://www.scielo.org.pe/pdf/liber/v22n2/a02v22n2.pdf

[15] S. Ibarra- Piza, S. Segredo-Santamaría, L. G. Juárez-Hernández, S. Tobón, "Estudio de validez de contenido y confiabilidad de un instrumento para evaluar la metodología socioformativa en el diseño de cursos," Revista espacios, vol. 39, no. 53, pp. 24, 2018, available: http://www.revistaespacios.com/cited2017/cited2017-24.html

[16] L. J. Cronbach, "Coefficient alpha and the internal structure of tests," Psychometrika, no.16, pp. 297-334, 1951.

[17] J. R. A. Santos, "Alfa de Cronbach: una herramienta para evaluar la fiabilidad de las escalas," Herramientas del oficio, vol. 37, no 2, 1999.

[18] I. Mavrou, "Análisis factorial exploratorio: cuestiones conceptuales y metodológicas," Revista Nebrija de Lingüistica Aplicada, no. 19, pp. 1-10, Nov. 2015. Disponible en: https://revistas.nebrija.com/revistalinguistica/article/view/283/248

[19] L. G. Juárez-Hernández, S. Tobón, “Análisis de los elementos implícitos en la validación de contenido de un instrumento de investigación,” Revista espacios, vol. 39, no. 53, pp. 23, 2017, available: https://www.revistaespacios.com/cited2017/cited2017-23.pdf

[20] J. A. Dorantes Nova, S. Tobón, “Instrumentos de evaluación: Rubricas Socioformativas," Praxis Investigativa ReDIE, vol. 9, no. 17, 2017, available: http://praxisinvestigativa.mx/assets/17_7_instrumentos.pdf

[21] C. S. Juárez-Lugo, K. Pichardo-Silva, M. C. Escoto-Ponce-de-León, E. Luna Montijo, "Confiabilidad y validez de la Escala de Estrategias de Aprendizaje acra en estudiantes universitarios del Estado de México," Investigación y práctica en psicología del desarrollo, vol. 1, pp. 261-268, 2015, available: https://revistas.uaa.mx/index.php/ippd/article/view/656

[22] Aniceto-Vargas, Luna-Nemesio, Rodríguez-Peralta. "Diseño y validación del instrumento: Gestión del Conocimiento en Proyectos formativos," (en proceso de publicación) Revista Educar, 2019. 
[23] A. B. Mirete-Ruiz, F. A. García-Sánchez, F. Hernández-Pina, "Cuestionario para el estudio de la actitud, el conocimiento y el uso de TIC (ACUTIC) en Educación Superior. Estudio de fiabilidad y validez," Revista Interuniversitaria de Formación del Profesorado, vol. 83, no. 29.2, pp. 75-89, 2015, available: https://dialnet.unirioja.es/servlet/articulo?codigo $=5249496$

[24] M. Á. Morata-Ramirez, F. P. Holgado-Tello, M. I. Barbero-García, G. Mendez, "Análisis factorial confirmatorio. Recomendaciones sobre mínimos cuadrados no ponderados en función del error Tipo I de Ji-Cuadrado y RMSEA," Acción psicológica, vol. 21, no.1, pp. 79-90, 2015, doi: http://dx.doi.org/10.5944/ap.12.1.14362

[25] J. F. Hair, W. C. Black, B. J. Babin, R. H. Anderson, Edition Multivariate Data Analysis, 7a ed. USA: Pearson Education, 2014.

[26] C. E. Bruna, V. A. Villarroel, D. V. Bruna, J. A. Martínez, "Experiencia de Diseño y Uso de una Rúbrica para Evaluar Informes de Laboratorio en Formato Publicación Científica," Formación Universitaria, vol. 12, no. 2, pp. 17-28, 2019, doi: http://dx.doi.org/10.4067/S0718-50062019000200017

[27] I. I. Neira Neaves, M. Ibáñez Cruz, H. M. López Oviedo, "Proceso de Validación de una Rúbrica Diseñada con el enfoque Socioformativo," in XIV Congreso Nacional de Educación Educativa (COMIE), San Luis Potosí, México, 2017, available: $\mathrm{http}: / /$ www.comie.org.mx/congreso/memoriaelectronica/v14/doc/2101.pdf

[28] V. Quesada Serra, G. Rodríguez Gómez, M. S. Ibarra Sáiz, "Planificación e innovación de la evaluación en educación superior: la perspectiva del profesorado," Revista de Investigación Educativa, vol. 35, no.1, pp. 53-70, 2017, doi: http://dx.doi.org/10.6018/rie.35.1.239261

[29] S. Tobón, Evaluación de competencias mediante rúbricas, México: CIFE, 2014, available: http://issuu.com/cife/docs/evaluaci_n_con_rubricas4

[30] C. Severiche-Sierra, E. Gómez-Bustamante, J. Jaimes-Morales, "La educación ambiental como base cultural y estrategia para el desarrollo sostenible," Telos: Revista de Estudios Interdisciplinarios en Ciencias Sociales, vol. 8 , no. 2, pp. 266-281, 2016, available: https://dialnet.unirioja.es/servlet/articulo?codigo $=5655393$ 